\title{
Costos de la reforestación semi-mecanizada y manual en potreros de la Chontalpa, México: un estudio comparativo
}

\author{
Michael R. Keyes Hennin ${ }^{1}$ \\ Javier Anduaga Muñoz ${ }^{2}$
}

RESUMEN

Los costos comparativos del establecimiento de una plantación semimecanizada vs una manual fueron calculados en una hectárea de potrero utilizando Tabebuia rosea y Cordia dodecandra. El método semi-mecanizado resultó más económico en un $17 \%$ y extremadamente rápido al compararlo con la plantación por métodos manuales. En general se aprecian diferencias en sobrevivencia y crecimiento que permiten concluir sobre la superioridad biológica y económica del método semi-mecanizado. Sin embargo, por la alta variabilidad, a pesar de que el método semi-mecanizado proporcionó mayores incrementos en altura que el método manual de plantación, estas diferencias no siempre fueron estadísticamente significativas.

PALABRAS CLAVE:

Métodos de reforestación, plantaciones forestales, sistemas silvopastoriles, Tabebuia rosea, Cordia dodecandra.

\begin{abstract}
The comparative establishment costs of a semi-mechanized vs manual plantation were calculated for one hectare of rangelands with Tabebuia rosea and Cordia dodecandra. The semi-mechanized plantation method was $17 \%$ less costly and much faster when compared with the manual method of plantation establishment. In general, better survival or seedling growth attest to the biological superiority of the semimechanized plantation method. However, due to the high variability, although the semi-mechanized plantation method offered improved height growth, these differences were not always statistically significant.
\end{abstract}

KEY WORDS:

Reforestation methods, forestry plantations, silvopastoral systems, Tabebuia rosea, Cordia dodecandra.

1 Departamento de Productos Forestales y Conservación de Bosques. Instituto de Ecología, A.C., Apdo. Postal 63, 91000 Xalapa, Ver., México.

2 Instituto Nacional de Geografía, Lima, Perú. Manuscrito recibido para su publicación el 26 de Septiembre de 1995 


\section{INTRODUCCION}

La mayoría de los países tropicales han utilizado plantas producidas en viveros, para mejorar tanto la composición específica, como la productividad de los bosques naturales. Entre los sistemas de plantación desarrollados se incluyen los siguientes tipos: 1) plantaciones de enriquecimiento en claros, cuando los árboles se plantan en sitios donde no hay árboles jóvenes, 2) plantaciones en hileras o grupos, cuando los árboles se plantan en hileras aclaradas o en grupos más o menos regulares, sobre un bosque parcialmente aclarado; 3) plantación cerrada, cuando se plantan suficientes árboles para asegurar por lo menos la cantidad prevista para la corta, sin ninguna participación de la regeneración natural (USFS, 1967). Las plantaciones forestales satisfacen diversas necesidades que van desde la protección, la recuperación y el control de la erosión hasta la conservación de cuencas. Por supuesto existen las plantaciones comerciales para la obtención de materia prima y hasta reforestación para fines escénicos.

De acuerdo con la experiencia y con el nivel tecnológico que posean los recursos humanos abocados a la reforestación, existe una variedad amplia de medios para alcanzar la diversidad de metas y extensiones de plantaciones en el trópico. En las plantaciones, ya sea con sistemas mecanizados o manuales, se usan generalmente plántulas producidas a raíz desnuda, estacas, o plántulas con cepellón (envase). En teoría también pueden realizarse plantaciones mediante siembra directa, no obstante que en la práctica la alta competencia de arvenses impide su realización en la mayoría de los casos. Las plantas a raíz desnuda han sido probadas exitosamente en México (Sánchez et al., 1988); sin embargo la plantación a raíz desnuda tiene el problema de ser muy susceptible a la desecación, aunque son menos costosas y menos voluminosas para su manejo en el campo y para su transporte al sitio. Intermedio en costos y también resistente se encuentra el material de estacas; la ausencia de suelo en este material facilita su manejo en el campo. Para evitar la mortalidad por desecación en transplantes tardíos, las estacas pueden ser cubiertas totalmente por el suelo durante el transplante; tal medio ha permitido inclusive el uso de herbicidas pre-emergentes bajo condiciones de vivero (Daniel et al., 1982). Con las plántulas producidas por cepellón se consigue un aumento en la sobrevivencia. A pesar de ello, existe la posibilidad de deformación, por lo que subsiste el dilema acerca de cuál es el método más conveniente.

Por ser el método más sencillo tecnológicamente y por evitar la desecación, el material producido en envase (cepellón) de gran tamaño actualmente es lo común en plantaciones forestales en una gran variedad de ambientes en América Latina. Las plántulas en envase grande se establecen con más facilidad que las producidos en envases pequeños; sin embargo, los costos de producción de envases de gran tamaño aumentan en proporción directa al tamaño de la plántula y volumen de suelo. Por lo anterior, y por costos tan altos de la plantación hecha a mano (manual) con envase grande, la tendencia actual es de utilizar la tecnología existente ya probada en otros ambientes, como es la técnica de raíz desnuda o bien, envases pequeños en bloques de tamaño manejable para un trasplantador en el campo.

Estos últimos envases consisten de un conjunto de más de cuarenta mini-envases; ya sean de plástico removibles de la rejilla o bien pueden llegar a ser desechables de cartón. Si bien la elección del material del envase grande puede ser lo más seguro para la sobrevivencia, seguramente los 
costos de producción, transporte y transplante son mayores $y$ hay que considerarlo, limitando la escala de la plantación y a veces la rentabilidad de la misma. Las ventajas económicas esperadas a partir de la producción de material vegetal dentro de envases se obtendrán sólo con el uso de envases de menor tamaño, los cuales son, paradójicamente, los menos convenientes para proporcionar alta sobrevivencia (Endean y Hocking, 1973). No solamente se requiere de mejoras en la tecnología para la producción de planta que sale del vivero, sino de un rango amplio de tecnologías para reforestar mayores extensiones (Keyes, 1996).

Durante muchos años se ha debatido sobre la necesidad de ganarle en velocidad a la deforestación, sin que prácticamente hoy en día existan evidencias para conocer el avance de la reforestación o los resultados concretos en México (Del Castillo, 1996); en esencia se ha olvidado el elemento fundamental: el costo de oportunidad entre la reforestación y otras opciones económicamente productivas. Los costos de la preparación mecanizada del sitio para plantaciones forestales son más bajos, pero incurren riesgos en suelos muy erosionables, pobres y con pendientes altas (Powers et al., 1988). Sin embargo, las ventajas de reducir los efectos de la competencia por agua y nutrientes hace atractiva una variedad de prácticas mecanizadas previas a la plantación (McNabb et al., 1993). Por otra parte, la limpieza de la plantación manual no economiza la mano de obra además de que se ha visto que limpieza con machete alarga el turno del jornalero (Endo y Wright, 1992). En México, actualmente una sociedad descapitalizada, se han planteado algunos esquemas financieros alternativos para facilitar la rentabilidad de las plantaciones forestales (Del Castillo, 1996).

\section{OBJETIVOS}

Con el objeto de mejorar la rentabilidad de las plantaciones forestales tropicales y darles mayor velocidad, se diseñó la presente investigación para probar por primera vez en México la preparación y transplante semi-mecanizado de arbolitos. El objetivo general fue comparar la variedad de técnicas necesarias para mejorar la rentabilidad y eficiencia en el establecimiento inicial de masas artificiales con la maquinaria ya disponible en el área de estudio. Específicamente los factores principales de analizar son la comparación de la rentabilidad o no del sistema con maquinaria y el método tradicional de preparación del sitio y transplante manual con dos especies comerciales.

\section{MATERIALES Y METODOS}

\section{Caracterización del área}

La presente investigación fue realizada en la región calido-húmeda denominada $\mathrm{La}$ Chontalpa, en el estado de Tabasco, geográficamente dentro de las coordenadas $93^{\circ} 33^{\prime}$ y $93^{\circ} 38^{\prime}$ de longitud oeste y $17^{\circ} 58^{\prime}$ hasta los $18^{\circ} 00^{\prime}$ latitud norte. Se ubica a 21 $\mathrm{km}$ oeste de la ciudad de Cárdenas dentro de las instalaciones del Centro Regional de Enseñanza y Capacitación Agropecuaria, Forestal y Acuícola del Sureste (CEICADES). La altitud va de los 7 a los 12 $\mathrm{m}$ sobre el nivel del mar. La estación meteorológica localizada en esa misma área revela un promedio de $2163 \mathrm{~mm}$ de precipitación pluvial, $1234 \mathrm{~mm}$ de evaporación y una temperatura media anual de $25.9^{\circ} \mathrm{C}$ en los últimos 27 años.

La vegetación natural de la región fue de selvas. A la fecha, esta región ha sido totalmente deforestada dejando en su lugar potreros y en el mejor de los casos bosques secundarios (acahuales) de menos de 12 años de edad. Las especies representativas de los acahuales del CEICADES son Guazuma ulmifolia, Gliricidia sepium, 
Tabebuia rosea, Pouteria campechiana, Trema micrantha, Eugenia capulli, Spondias mombin, Diphysa rubinoides, Phoebe sp., Sapindus saponaria, Bursera simaruba, Lonchocarpus cruentus y Colubrina arborescens.

El suelo donde se desarrolló el experimento, previamente forestal y actualmente con pastizales y acahual, está clasificado como vertisol crómico en el sistema de FAO-UNESCO, con permeabilidad lenta. Fisiográficamente se ubica en las planicies del Golfo de México de formación reciente. Se encontraron cuatro horizontes: dos "A" y dos "C". El A11 va de 0 a $35 \mathrm{~cm}$, el $A 12$ de 35 a $85 \mathrm{~cm}$, el C1g de 85 a $145 \mathrm{~cm}$ y el C2g de 145 a 200 $\mathrm{cm}$. Este suelo es de pobre a muy pobre en materia orgánica, el nitrógeno total es moderadamente pobre y el fósforo es mediano a pobre. El calcio y magnesio es alto en todo el perfil; el potasio es bajo en todo el perfil y del aluminio únicamente existen trazas (Trujillo y Palma, 1988). Corresponde a la serie Limón de textura arcillosa. Los colores en húmedo son café amarillento en la superficie y presenta motas abundantes rojo-amarillentas. La estructura es columna y en bloques fuertes.

\section{Especies utilizadas}

Las especies forestales que se utilizaron son: Tabebuia rosea (Bertol.) DC. conocida localmente como "maculís" y Cordia dodecandra A. DC. conocida como "siricote". Con este material vegetal se probaron los sistemas de plantación con material producido tanto a raíz desnuda como en envase con cepellón. Las plántulas de maculís tenían ocho meses de edad con $92 \mathrm{~cm}$ de altura en promedio, provenientes del mismo municipio, mientras que las de siricote fueron traídas desde el municipio de Felipe Carrillo Puerto en el estado de Quintana Roo teniendo cinco meses de edad y $29 \mathrm{~cm}$ de altura en promedio al momento de la plantación.

\section{Diseño experimental}

Se ensayaron cuatro tratamientos principales:

I. semi-mecanizado a raíz desnuda

II. semi-mecanizado con cepellón

III. manual a raíz desnuda y

IV. manual con cepellón

En cada uno de estos tratamientos se probaron dos especies con potencial comercial. También se probaron estas tecnologías en las dos condiciones ecológicas prevalecientes en el área de estudio. Para lograr lo anterior, fue localizado en un bosque secundario (acahual) adyacente a un potrero, de tal forma que en un reducido espacio con similitud de suelos, se presentan estos dos tipos de vegetación aptos para reforestación en la región: potrero (denominado campo abierto) y bosque secundario (denominado dosel protector). Los resultados de este estudio son presentados en conjunto con otros sobre crecimiento temprano en plantaciones semi-mecanizadas en el trópico. Se utilizó un diseño de Bloques Completamente Aleatorio en cada una de las dos condiciones ecológicas: campo abierto y dosel protector. Para el caso del maculís, se realizaron cuatro repeticiones por tratamiento, con 12 plántulas dentro de cada repetición, sumando un total de 384 plántulas. Para el siricote se utilizaron tres repeticiones por tratamiento y seis plántulas dentro de cada repetición, de modo que de los cuatro tratamientos en total fueron 144 plántulas. Los tratamientos fueron distribuidos al azar en cada una de las condiciones, formando finalmente una plantación en hileras empezando en campo abierto y terminando dentro del bosque en las brechas bajo dosel protector. Antes de 
traer la maquinaria o los brecheros, con apoyo de una brújula se marcaron con estacas las hileras siguiendo líneas paralelas.

\section{Preparación del terreno}

Se inició el tratamiento denominado "semi-mecanizado" en dicho Centro Experimental que consistía en usar un tractor de oruga, el cual abrió las brechas en el bosque secundario con un ancho aproximado de 2.5 metros, que es el ancho de la pala frontal del tractor. El empleo de este tractor no fue necesario para la preparación del terreno en el tratamiento "semi-mecanizado" en campo abierto. En tal caso el tractor de llantas neumáticas realizó un chapeo con el implemento "desvaradora", previo al transplante. Se aprovechó la presencia del ayudante del tractor en el sitio quien además de ir adelante cortando con machete algunas ramas peligrosas también prestaba apoyo bajando periódicamente los arbolitos al suelo y posteriormente acomodándolos a un lado de la brecha. Para la plantación denominada "manual" bajo el dosel protector y en el campo abierto, las brechas fueron abiertas manualmente con machete y las plantas fueron traídas desde el camión a mano y colocadas al lado de los hoyos.

\section{Plantación manual}

Mediante el procedimiento manual se logró una apertura de brecha de 2.5 metros en el acahual. Posteriormente con la ayuda de una "cava hoyos" de operación manual, utilizada comúnmente en la región, un trabajador hacía la apertura de cepas a 20-28 cm de profundidad, otro trabajador en seguida depositaba las plántulas acomodándolas bien, tapándoles con suelo y compactando con el pie. Ya sea a raíz desnuda 0 con cepellón, las labores culturales de limpieza fueron también realizadas con machete. Posterior al transplante se realizaron dos limpiezas de las plantaciones en potrero y en acahual.
Para fines comparativos del análisis de costos que se reporta más adelante, se tomó el promedio de estas dos condiciones ecológicas. Cabe señalar que la apertura de brechas fue más lenta y las limpiezas más rápidas en el acahual.

\section{Plantación semi-mecanizada}

En el tratamiento denominado aquí como "semi-mecanizado", se utilizó la tecnología disponible que consiste en usar un tractor de oruga marca Caterpillar D-4, el cual abrió las brechas en el bosque secundario; para efectuar el transplante se diseñó un implemento montado sobre un tractor convencional marca Ford, modelo 6600. El método denominado "manual" es el comúnmente utilizado en México y su procedimiento se explica más adelante. Por cada uno de los dos sistemas de transplante se utilizaron dos variantes del sistema de producción de plántula: raíz desnuda y cepellón.

Se realizó el transplante con la ayuda de un tractor de llantas neumáticas provisto de un levante hidráulico con enganche en tres puntos. Sobre este levante se colocó la barra "porta implementos" y en la mitad de la misma se insertó una reja o arado de doble vertedera modificada de tamaño pequeño, la cual al ser bajada al suelo, producía un corte con la forma de "flecha de reja". La modificación de la reja fue necesaria y consistió en soldar placas de fierro desde la base de las alas hasta la barra central, a fin de que el suelo al momento de ser levantado cayera sobre la parte delantera de la reja permitiendo una mejor calidad en la apertura de hoyos (Fig. 1). Una vez que la reja profundizaba en el suelo de 20 a $25 \mathrm{~cm}$, el operador procedía a levantarla avanzando ligeramente. Esta operación se sincronizaba con el fin de producir una apertura adecuada para poder depositar la plántula, ya sea a raíz desnuda o cepellón por parte de los plantadores quienes iban siguiendo al tractor para efectuar el entierre manual correcto de la 
plántula con ayuda de su pie. Debido a que la apertura de hoyos es más rápida, es posible que dos o más obreros puedan acompañar al operador del tractor convencional para un aprovechamiento más eficiente de la mano de obra. Trabajando así, la plantación mecanizada tenía un promedio de distancia entre hileras de cinco y de dos metros entre plántula y plántula.

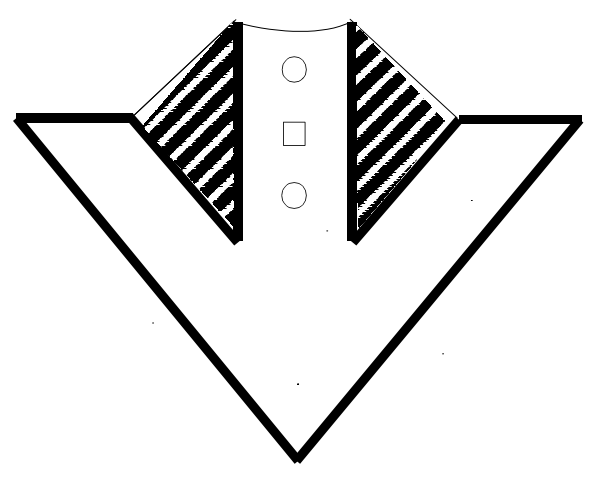

Figura 1. Reja o arado de doble vertedera modificada con la adición de dos placas soldadas a las alas

Posterior al transplante se realizaron dos limpiezas bajo este sistema de plantación tanto en potrero como en acahual. Para estas labores culturales en la plantación semi-mecanizada, en el acahual se usó el machete. En contraste, en la plantación manual se usó la desvaradora del tractor agrícola principalmente en el potrero. No obstante lo anterior, fue necesario utilizar menos de un jornalero con machete para limpiar la faja de malezas que deja en pie este cuchillo circular. Esta limpieza, principalmente entre planta y planta, promediaba menos de $30 \mathrm{~cm}$, debido a que el tractor alcanzaba a limpiar casi el 100\% alrededor de las plantas y se desviaba luego para evitar dañarlas al cercar cada "matita". Como consecuencia, resultó muy rápida la escasa limpieza manual requerida en los alrededores de estas plantas en el potrero. Para fines comparativos del análisis de costos, al igual que en el caso de la plantación manual, se toma en cuenta el promedio de estas dos condiciones ecológicas. Cabe recordar que la apertura de brechas fue más rápida en el potrero.

\section{Fecha de plantación y mediciones}

La plantación se realizó en el mes de julio de 1988, cuando la humedad de los suelos alcanzaba su Capacidad de Campo. Una semana después se realizó una medición preliminar con la finalidad de investigar si hubiera un sesgo después de la selección aleatoria que pudiera favorecer a alguno de los tratamientos. No hubo diferencias iniciales en el tamaño de las plantas entre tratamientos. Las evaluaciones reportadas en el presente estudio fueron tomadas en el mes de marzo de 1989, representando así un crecimiento de ocho meses.

\section{Variables evaluadas}

Los costos de la plantación fueron contabilizados para los dos sistemas: semimecanizado y manual en el acahual. El costo de hora-máquina utilizado en esta comparación corresponde con los precios de arrendamiento utilizado por el gobierno para proyectos sociales de reforestación. Posteriormente, estos costos fueron calculados en dólares (US\$) para facilitar comparaciones con otras monedas y para facilitar la interpretación a pesar de los cambios por devaluaciones del peso. De igual manera para fines comparativos, los datos obtenidos en el trabajo de aproximadamente $6,700 \mathrm{~m}^{2}$ se extrapolaron a una hectárea. En el cuadro comparativo de costos se presenta un promedio de las condiciones de potrero y bosque secundario. En los dos casos la plantación es equivalente a 1,000 plántulas/ha. Las variables evaluadas luego de ocho meses 
de crecimiento de la plantación fueron sobrevivencia e incremento en altura, a los ocho meses de haber efectuado el trasplante.

\section{Análisis estadístico e hipótesis}

Se realizó un análisis de varianza (ANOVA) previo al período de crecimiento y también posterior. El análisis de varianza aplicado al inicio del experimento no indicó diferencias significativas para las dos especies ni para las dos variables de altura inicial en cualquiera de los tratamientos de semi-mecanización, manual, dosel protector o campo abierto. Por lo anterior, los resultados que se presentan en este reporte de las mediciones tomadas a los ocho meses de transplante, indican diferencias por el comportamiento de las plántulas y no por factores ajenos al experimento. El análisis estadístico se hizo con el objeto de probar la siguiente hipótesis nula: el crecimiento de plántulas en el sistema de plantación manual es superior comparado con el sistema semi-mecanizado. Es presentada aquí la estadística "F" proveniente del análisis de varianza para la variable de sobrevivencia.

Por lo anterior, además del análisis tipo ANOVA, también se efectuó un análisis no-paramétrico para determinar si la variabilidad entre promedios del sistema semi-mecanizado comparado con el trasplante manual fuera estadísticamente diferente. Se indica si efectivamente hubo diferencias entre los promedios de altura utilizando la prueba de "U" de Mann-Whitney (no paramétrica) por el período de crecimiento posterior al trasplante. Por ello se relacionaron la estadística "U" con los valores estándar de "z" provenientes de la curva normal estándar, mismos que tuvieron que ajustarse al criterio mínimo de 0.05 como nivel de probabilidad para los fines del presente ensayo.

\section{RESULTADOS Y DISCUSION}

Comparación de los costos de los sistemas de plantación semi-mecanizado contra el sistema manual

Los resultados presentados en la Tabla 1 muestran la ventaja económica del sistema semi-mecanizado comparado con el sistema de plantación manual. Mientras la plantación hecha básicamente a mano alcanzó un costo de US\$217/ha, los costos para la plantación semi-mecanizada fueron tan sólo US\$178/ha. Esta diferencia de US\$ $39 /$ ha representa un ahorro del $17 \%$ en los costos de establecimiento y mantenimiento durante el primer año. El costo que se alcanzó en el método semi-mecanizado podría reducirse aún más, usabdo un tractor agrícola provisto de una pala frontal para hacer la preparación requerida del sitio. Por otra parte, se alcanzó a sembrar un promedio de 880 plántulas/hora en el sistema semi-mecanizado, lo cual incluye tanto la apertura de hoyo como el trasplante propiamente dicho "tapa pie". Las velocidades de trasplante obtenidas están dentro de los límites de rentabilidad expresados por McKenzie et al. (1984). Para mayores rendimientos por unidad de tiempo se requiere de maquinaria especializada. Por ejemplo, Richardson (1989) trabajando en sitios planos reportó velocidades de entre 1,875 a 2,500 plántulas por hora de trabajo con brigadas de ocho personas con la maquinaria de trasplante, fertilización y aplicaciones químicas.

Los costos presentados reflejan la optimización de los recursos que estaban disponibles en La Chontalpa, en el tiempo en que se realizó el estudio. Por ejemplo, para este ensayo, el costo para la compra y la soldadura para modificar la flecha fue alrededor de US\$ 65, que es lo que realmente se tuvo que pagar para complementar el esquema de plantación 
Tabla 1. Costos comparativos (en dólares de Estados Unidos) por hectárea del establecimiento de una plantación forestal bajo los sistemas semi-mecanizado y manual de un potrero enmontado por cinco años

\begin{tabular}{|c|c|c|c|c|c|}
\hline & \multicolumn{3}{|c|}{ SEMI-MECANIZADO } & \multicolumn{2}{|c|}{$\overline{\text { MANUAL }}$} \\
\hline Actividades & Hora & Jornales $^{1}$ & Costos & Jornales $^{1}$ & Costos \\
\hline $\begin{array}{l}\text { Localización, trazo y marcado } \\
\text { inicial de brechas }\end{array}$ & - & 2.2 & 10.9 & 2.2 & 10.9 \\
\hline $\begin{array}{l}\text { Apertura de brechas y hoyos } \\
\text { con Caterpillar D- } 4^{2} \\
\text { ayudante de tractorista }\end{array}$ & $\begin{array}{r}- \\
3.0 \\
-\end{array}$ & $\begin{array}{r}1.0 \\
- \\
-\end{array}$ & $\begin{array}{r}5.0 \\
50.4 \\
5.0\end{array}$ & $\begin{array}{r}19.0 \\
- \\
-\end{array}$ & $\begin{array}{r}94.2 \\
- \\
-\end{array}$ \\
\hline Estacado y marcado de hoyos & - & 0.5 & 2.5 & 0.5 & 2.5 \\
\hline Traslado de plántulas & - & 1.2 & 6.0 & 2.0 & 9.9 \\
\hline $\begin{array}{l}\text { Trasplante } \\
\text { con tractor } \\
\text { estacado final }\end{array}$ & $\begin{array}{r}- \\
3.0 \\
-\end{array}$ & $\begin{array}{r}2.2 \\
- \\
0.5\end{array}$ & $\begin{array}{r}10.9 \\
21.6 \\
2.5\end{array}$ & $\begin{array}{r}4.0 \\
- \\
0.5\end{array}$ & $\begin{array}{r}19.8 \\
- \\
2.5\end{array}$ \\
\hline $\begin{array}{l}\text { Primera limpia } \\
\quad \text { tractor con desvaradora }{ }^{3}\end{array}$ & $4 . \overline{-}$ & $\begin{array}{r}0.6 \\
-\end{array}$ & $\begin{array}{r}3.0 \\
29.5\end{array}$ & $\begin{array}{r}7.5 \\
-\end{array}$ & $\begin{array}{r}37.2 \\
-\end{array}$ \\
\hline $\begin{array}{l}\text { Segunda limpia } \\
\text { tractor con desvaradora }\end{array}$ & $3 . \overline{7}$ & $\begin{array}{r}0.8 \\
-\end{array}$ & $\begin{array}{r}4.0 \\
26.6\end{array}$ & $\begin{array}{r}8.0 \\
-\end{array}$ & $\begin{array}{r}39.7 \\
-\end{array}$ \\
\hline $\begin{array}{l}\text { Labores culturales totales, } \\
\text { jornales u horas/ha }\end{array}$ & 13.8 & 10 & & 33.7 & \\
\hline COSTOS TOTALES / HA & & & $\$ \$ 177.9$ & & \$216.7 \\
\hline
\end{tabular}

1 Costo de Jornal promedio en La Chontalpa, Tabasco, México = US\$4.96/día.

2 Tractor de orugas Caterpillar D-4 = US\$16.8/hora; tarifa de arrendamiento por el Gobierno Estatal de Tabasco para trabajos agropecuarios en La Chontalpa, incluyendo el salario del operador

3 Tractor de ruedas Ford $6600=$ US $\$ 7.20 /$ hora; tarifa de arrendamiento por el Gobierno Estatal de Tabasco para trabajos agropecuarios en La Chontalpa incluyendo el salario del operador. 
semi-mecanizada. En estos ensayos se utilizó maquinaria ya existente y con adaptaciones con costos mucho menores que los promedios de los costos reportados en US $\$ 10,000$ para las unidades remolcadas por tractores (McKenzie et al. 1984). Para este análisis no se consideró el costo de la plántula, ya que éstas son comúnmente regaladas para cualquier reforestación en México y para el presente experimento fueron efectivamente regaladas, al igual que para el caso de la plantación reportada por Rojas (1995) en el estado de Veracruz, México. Para ningún tratamiento hubo necesidad de fumigaciones o fertilizantes. Además, fueron optimizados los costos por haber transportado las plántulas con tractor desde el camión hasta las brechas, previo a la apertura de los hoyos con el paso del tractor; de haber tenido un remolque con el tractor se hubiera ahorrado más tiempo. Por otra parte, en este ensayo podría existir un costo oculto para la reconstrucción del levantamiento hidráulico del tractor. Se considera que el levantamiento intermitente de la barra porta-implementos podría implicar un gasto adicional por la frecuente reposición de empaques. Sin embargo, los mecánicos especializados sugirieron que este costo no debía incluirse dentro de este análisis por ser insignificante con respecto al costo total por la operación de la maquinaria en la región de estudio.

Los costos de los dos sistemas de plantación son iguales 0 menores comparados con algunos otros reportes. Rojas (1995) en una plantación cerca de el sitio de este estudio reportó costos de establecimiento de US\$236.3/ha para Cedrela odorata. En otros ambientes como en EUA los costos son mayores. Principalmente esto se debe al alto costo allá, que se aplica para la mayor parte de las actividades del campo, sobre todo para mano de obra, maquinaria y plántulas. Mientras Chapman y Allen (1978) señalan que las plantaciones debían costar alrededor de US $\$ 1,000 /$ ha, habría que considerar los costos relativos en los diferentes ambientes socio-económicos. En el presente ensayo, como se menciona anteriormente, no está contemplado el costo de la plántula, tampoco hubo necesidad de fumigaciones o fertilizantes.

\section{Sobrevivencia y crecimiento}

La sobrevivencia de maculís en los cuatro tratamientos y para las dos condiciones ambientales evaluadas no presentó diferencias significativas; todos los tratamientos produjeron una alta sobrevivencia que fue de $95 \%$ a $100 \%$. Estos promedios tan altos para maculís, aunque indican una ligera ventaja para el sistema de trasplante semi-mecanizado, no fueron estadísticamente significativos. Los resultados de este estudio concuerdan con las conclusiones vertidas por Ladrach (1982) en su estudio al término de un año; no siempre hay diferencias estadísticamente significativas en sobrevivencia, por el alto nivel de variabilidad, pese a que estas tendencias se manifiestan más adelante durante fases subsecuentes en la plantación.

La sobrevivencia de siricote en el tratamiento principal de semi-mecanizado fue $12.8 \%$ mayor que en el sistema manual de plantación. El análisis de varianza para la variable sobrevivencia de siricote bajo el dosel protector y campo abierto mostraron diferencias significativas para uno de los tratamientos factoriales por lo menos (Tabla 2). En el tratamiento de cepellón, la sobrevivencia fue también excelente, obteniendo el $100 \%$ de sobrevivencia tanto para los tratamientos semi-mecanizados con envase y manual con envase. Dos semanas después de haber efectuado el trasplante, se presentó una sequía de más de trece días en la zona de La Chontalpa. No fueron regadas las plántulas, para ver si los extremos que se pudieran presentar afectaban en forma diferente a cada método de trasplante en cada una de las 
condiciones ecológicas. La sobrevivencia global, por ende, bajó a $33 \%$ en el tratamiento semi-mecanizado a raíz desnuda y $22 \%$ en el tratamiento manual a raíz desnuda. Esta baja sobrevivencia para el siricote bajo el sistema de raíz desnuda se debe al doble trasplante que sufrieron las plantas. Se debe destacar que tal mortalidad no se debió al efecto en sí de los tratamientos principales del experimento, sino a un doble manipuleo y al traslado que sufrieron estas plántulas al transportarlas a los ocho meses de edad desde un vivero de Quintana Roo; esto además de haberse presentado la sequía mencionada. No obstante lo anterior, el promedio de sobrevivencia de siricote, combinando las condiciones de raíz desnuda y cepellón fue mejor tanto para el trasplante semimecanizado como para el manual como se puede apreciar en la Tabla 2.

La variable de altura presenta algunas tendencias similares a las de sobrevivencia. Para la variable incremento en altura, se ratificó la superioridad del método semimecanizado para la especie maculís en ambas condiciones ecológicas, alcanzando un incremento de $51.1 \mathrm{~cm}$, contra sólo 42.8 $\mathrm{cm}$ en los métodos manuales (Tabla 3).

El análisis de varianza para las variables de incremento en altura para maculís indicó que por lo menos un tratamiento fue significativo. Hubo también interacción en ambas variables entre las dos condiciones ecológicas y los tratamientos semimecanizado y manual. Para el siricote el crecimiento en altura fue mejor en el sistema semi-mecanizado y el análisis de varianza mostró $F=17.20$ para los incrementos en altura con probabilidades de $>0.001$ para indicar que por lo menos uno de los tratamientos fue significativamente diferente.
La prueba de Mann-Whitney, una de las más versátiles pruebas no paramétricas, indica que hubo superioridad en el crecimiento bajo el sistema semimecanizado. Esta afirmación es válida si se conjuntan las dos condiciones ecológicas. Viendo por separado los tratamientos principales, siempre hubo menor crecimiento en altura bajo el sistema de trasplante manual; no obstante que el crecimiento fue casi siempre mayor para la plántula producida por cepellón (envase).

El sistema semi-mecanizado ofreció incrementos en altura $25.9 \%$ superiores comparado con el crecimiento bajo el sistema manual. Mayores incrementos (32.2\%) fueron encontrados para maculís con el sistema semi-mecanizado y $19.6 \%$ más en crecimiento fue observado en los ensayos de siricote. Los resultados de Tabebuia rosea (que no sufrió daños en el trasplante) parece comportarse de manera muy similar a las demás especies forestales tropicales en esta etapa temprana después del trasplante (Tabla 4). Los promedios obtenidos de incrementos por el efecto del uso de maquinaria se ven conservadores (Tabla 4), no obstante que Lamberth (1986) encontró solamente $24.6 \%$ incrementos mayores en altura con control profundo de pastos en potreros a los dos años de medición.

Las ventajas biológicas de la mecanización no solamente son obvias, sino que existe literatura amplia que confirma estos hechos. La competencia por agua, nutrientes y luz entre arbolitos y malezas como pastos en los potreros- es feroz (Cannon et al., 1984) en las etapas iniciales. El control de esta competencia de pastos en plantaciones forestales es necesaria hasta el cierre de las copas y por consiguiente, los costos de limpieza se reducen al mínimo después. Existen numerosas investiga- 
Tabla 2. Análisis de varianza para la sobrevivencia bajo dos sistemas contrastantes de producción de plántula y plantación combinando la plántula producida a raíz desnuda y en cepellón.

\begin{tabular}{||l|r|r|r|r|c||}
\hline \multicolumn{1}{||c|}{ SISTEMA } & $\begin{array}{c}\text { SEMI- } \\
\text { MECANIZADO }\end{array}$ & MANUAL & $\begin{array}{c}\text { GRADOS DE } \\
\text { LIBERTAD }\end{array}$ & F & Pr $>$ F \\
\hline \multicolumn{7}{|c||}{ Cordia dodecandra (siricote) } \\
\hline Dosel protector & 50.0 & 48.6 & 11 & 11.40 & 0.0069 \\
\hline Campo abierto & 74.2 & 62.7 & 11 & 3.66 & 0.0483 \\
\hline Promedio1 & 6.24 & 55.3 & & & \\
\hline \multicolumn{7}{|c|}{ Tabebuia rosea (maculís) } & 0.36 & 0.7834 \\
\hline Dosel protector & 96.1 & 97.7 & 15 & 5.40 & 0.0211 \\
\hline Campo abierto & 98.8 & 95.7 & 15 & & \\
\hline Promedio & 97.45 & 96.5 & & & \\
\hline
\end{tabular}

1 Promedio de dos condiciones ambientales combinadas; campo abierto y dosel protector

Tabla 3. Crecimiento en altura bajo dos sistemas contrastantes de producción de plántula y de plantación

\begin{tabular}{|c|c|c|c|}
\hline SISTEMA & $\begin{array}{c}\text { SEMI- } \\
\text { MECANIZADO } \\
\text { PROMEDIO }\end{array}$ & $\begin{array}{l}\text { MANUAL } \\
\text { PROMEDIO }\end{array}$ & $\begin{array}{c}\text { NIVEL DE } \\
\text { SIGNIFICANCIA }^{1}\end{array}$ \\
\hline \multicolumn{4}{|c|}{ Cordia dodecandra (siricote) } \\
\hline Raíz desnuda & $21.2 \quad(8.31)^{3}$ & $16.0 \quad(9.64)$ & NS \\
\hline Cepellón & $81.0 \quad(9.64)$ & $69.5 \quad(5.75)$ & ** \\
\hline Promedio $^{2}$ & 51.1 & 42.8 & ** \\
\hline \multicolumn{4}{|c|}{ Tabebuia rosea (maculís) } \\
\hline Raíz desnuda & $19.8 \quad(4.37)$ & $10.3 \quad(5.88)$ & ** \\
\hline Cepellón & $22.8 \quad(4.56)$ & $21.8 \quad(7.09)$ & NS \\
\hline Promedio $^{2}$ & 21.3 & 16.1 & * \\
\hline
\end{tabular}

1 * indica que la probabilidad de error es menor que " $=0.05$; prueba de Mann-Whitney

** indica que la probabilidad de error es menor que " $=0.01$; prueba de Mann-Whitney

2 promedios sumando ambos tipos de plántulas; cepellón y raíz desnuda

3 números entre paréntesis corresponden a la desviación estándar 
Tabla 4. Promedios de incrementos en altura por los efectos de mecanizado sobre preparación del sito manuales en plantaciones tropicales a la edad de dos años y menores

\begin{tabular}{||l|c|l||}
\hline \multicolumn{1}{||c|}{ ESPECIE } & $\begin{array}{c}\text { INCREMENTO ALTURA }{ }^{1} \\
(\%)\end{array}$ & \multicolumn{1}{|c||}{ REFERENCIA } \\
\hline Cordia dodecandra & 19.6 & Keyes y Anduaga. 1997 \\
\hline Cupressus lusitanica & 70.3 & Ladrach, 1982 \\
\hline Eucalyptus globulus & 24.6 & Lamberth, 1986 \\
\hline Eucalyptus grandis & 34.6 & Ladrach, 1982 \\
\hline Eucalyptus teticornis & 101.0 & Retrepo, 1990 \\
\hline Pinus oocarpa & 9.1 & Ladrach, 1982 \\
\hline Tabebuia rosea & 134.0 & Retrepo, 1990 \\
\hline Tabebuia rosea & 32.2 & Keyes y Anduaga, 1997 \\
\hline \hline \multicolumn{2}{|c|}{ 25.9 } & LROMEDIO DE INCREMENTO \\
\hline Presente Estudio & 62.3 & Global \\
\hline \multicolumn{2}{|c|}{53.2} \\
\hline \hline
\end{tabular}

ciones sobre esta etapa crítica (Ladrach, 1982; Retrepo, 1990; Endo y Wright, 1992).

El control de competencia, es de los elementos de mayor peso a enfrentar en las plantaciones comerciales provenientes de potreros (Endo y Wright, 1992). Entonces, es común encontrar el doble de incremento en altura bajo un sistema eficiente de control de malezas en esta etapa de establecimiento (Retrepo, 1990). También Cannon (1982) reportó incrementos promedios de $83 \%$ usando el arado y dos pasos de rastrillo comparado con la preparación manual del sitio. Es claro que los mejores resultados se den con una combinación entre varias tecnologías. Las ventajas biológicas de la mecanización se mostraron como ventajas claras para el crecimiento inicial sobre el método manual, también por la eliminación de competencia radicular por presencia de pasto en los potreros. A la hora de establecer la plantación, la reja del tractor después de penetrar desplazaba un volumen de 500 a $1,650 \mathrm{~cm}^{3}$ de suelo al levantarse. Comparativamente en la cepa hecha con cava-hoyos el área preparada para la plántula fue de tan sólo 200 a $380 \mathrm{~cm}^{3}$, lo cual efectivamente, no dejó mucho substrato libre de competencia para el desarrollo radicular posterior al trasplante. 
Las tendencias sobre el crecimiento promedio y sobreviencias son claras en estos ensayos. El análisis de varianza indica superioridad de alguno de los tratamientos en las variables de sobrevivencia y altura. La sobrevivencia general con el sistema semi-mecanizado también está favorecido por la mecanización de labores culturales. No obstante lo anterior, de acuerdo con las variables analizadas estadísticamente, no siempre se puede afirmar que alguno de los tratamientos ensayados ofrezca superioridad, sobre todo en experimentos con muchas variables y desde luego, con alta variabilidad entre las plántulas lo que evita diferenciar estadísticamente entre los crecimientos por los tratamientos principales. Esta variabilidad, no deseada es difícil de evitar, ya que se trata de poblaciones no seleccionadas genéticamente. Es importante señalar que en el presente análisis sí fue posible hacer evidentes las ventajas del sistema semimecanizado sobre el manual con base en el crecimiento de plántulas, a pesar de la complejidad del experimento. Además de la variabilidad del germoplasma y de las diferencias en sobrevivencia, las repuestas a los diferentes tratamientos de condiciones ecológicas, la especie y el sistema de plántula (p.e. cepellón o raíz desnuda) aumenta la variabilidad. Estos resultados concuerdan además con los presentados en la literatura, considerando que en todos los estudios anteriores que se citan, la evaluación se ha hecho antes de que las plantaciones alcancen los dos años de edad. Los autores señalan que las tendencias iniciales de superioridad de la mecanización aumentan con la edad de la plantación (Ladrach, 1982; Lamberth, 1986).

El control de malezas puede constituir uno de los costos más altos en el primer año de una plantación. El control químico de malezas se ha demostrado en Colombia, como más efectivo que el control manual (Endo, 1992; Endo y Wright, 1992). Un problema encontrado es, que la presencia de muchas especies de "hoja ancha" son muy susceptibles a los herbicidas igual que las especies forestales, lo cual parece una limitante para el control químico de malezas en los bosques secundarios. Si el productor no cuenta con herbicidas, el arado y doble rastrillo han dado mejores resultados que el deshierbe por completo. El efecto del arado - el repique de un área de un metro cuadrado son similares (Osorio, 1988).

En general, se encontró que la velocidad para realizar labores como la limpieza son semejantes a los que reportan estos autores y mayores a los que reportó Rojas (1995). En el presente estudio las limpiezas en el acahual fueron más lentas comparadas con las acciones combinadas del tractor con la desvaradora y limpieza manual con machete para terminar el chapeo de las islas de malezas que estuvieron fuera del alcance de la desvaradora. Se encontró también que el tractor con desvaradora no entraba al lado de la hilera de arbolitos en el bosque secundario. Una manera de mecanizar estas limpiezas bajo la sombra protectora podría ser el uso de la desmalezadora tipo "weed eater". Estas desmalezadoras con motores pequeños y con cabezas cortantes de cuchillo o hilo han podido ser utilizadas posteriormente con éxito en otras plantaciones en La Chontalpa, no obstante que no estuvieron disponibles a la hora que se realizó este estudio. La ventaja que presenta esta herramienta sobre los herbicidas es que su efecto es directo, selectivo y más confiable en las temporadas de lluvias.

Para las plantaciones comerciales el uso de tecnología es recomendable. Endo y Wright (1992) encontraron que la limpieza con machete no economiza en mano de obra comparada con la aplicación de herbicidas en franjas, además de que se retrasa en cerrar la copa hasta los 18 ó 22 
meses de edad. Evans (1982) afirma que la mecanización para la mayoría de las plantaciones industriales es ampliamente utilizada en las zonas tropicales alrededor del mundo, sobre todo en las operaciones que consumen mucho tiempo y mano de obra, por ejemplo, para fines de preparación del sitio, excavación de hoyos, trasplante y aplicación de fertilizantes y herbicidas. El uso de la desvaradora (cuchillo circular remolcado por el tractor e impulsado por la toma de fuerza) resultó de gran importancia práctica para mantener las plantaciones del experimento. A pesar de que se use una desvaradora, siempre se va a requerir de limpiezas más finas entre planta y planta. Por ello, la limpieza con machete o aplicación de herbicidas son las opciones de mayor uso. Los autores se reservan su opinión respecto a la rentabilidad del uso de maquinaria pesada en muchas plantaciones forestales, especialmente si se tiene que comprar la maquinaria para tales fines. El uso efectivo de los materiales de uso común en el ámbito agropecuario y a la mano, tal vez sea de los factores que se deba dar mayor peso al elegir los métodos para realizar una reforestación artificial.

No siempre se puede costear un tractor de orugas. Como medida práctica es recomendable usar el mínimo de maquinaria, siempre y cuando se esté seguro de que hay personal entrenado y refacciones para sostener su uso. Igual o peor que en campo inundado y resbaloso la vegetación secundaria no siempre permite que avance el tractor convencional. Existen, desde luego en los ambientes arroceros, canastillas para cubrir las llantas e inclusive la práctica de usar doble rodada cuando los campos son resbalosos. En este sentido un tractor convencional puede entrar en un espeso bosque secundario si cuenta con el auxilio de personal de apoyo para abrir la brecha manualmente siempre y cuando no se atasque; de lo contrario, puede ser peligroso el uso de maquinaria liviana que se atasque en vegetación densa sin el auxilio de "brecheros" y una caseta de protección para el operador.

En países en vías de desarrollo se encuentra maquinaria tanto liviana como pesada para la agricultura o para la construcción. En estos casos por falta de conocimientos y herramientas básicas los forestales han perdido oportunidades excelentes para avanzar en la reforestación como un proyecto de interés comunitario. El caso común es el uso de la trasplantadora. En los países más industrializados en donde existen una variedad muy amplia de herramientas, el cuchillo circular sigue siendo "la ley"; en el trópico la trasplantadora es aún una incógnita. No obstante, puede considerarse al tractor como lo básico, y los implementos auxiliares pueden ser sustituidos o mejorados según el caso. Por ejemplo, con el simple arrastre de uno o dos timones, también conocido como subsoleo (subsuelo), se logra hacer el surco continuo parecido o mejor que las máquinas trasplantadoras comúnmente utilizadas en otros ambientes. En Colombia, la mecanización de la preparación del sitio ha sido exitosa y se usa un paso de subsolado con dos o tres pasos del rastrillo. Endo (1992) reporta la preparación del sitio en 5 horas/ha con tractor, semejante a los resultados obtenidos es este estudio.

Como una aclaración pertinente, sobre las bondades de los métodos mecánicos de plantación, en Chile se compararon dos tipos de maquinaria con la plantación manual, sobre terrenos arenosos. Los resultados logrados por las máquinas en jornadas de ocho horas fueron de 3 a 4.5 hectáreas con plantación a $2 \times 2$ metros. La plantación chilena semi-mecanizada resultó superior de 8 a 12 veces comparada con la plantación manual, reduciendo a la vez los costos en un $50 \%$ en comparación con los de la labor manual (Zanartu, 1968). No obstante, desde un punto de vista teórico, 
los costos de sistemas mecanizados deben ser semejantes o un poco superiores a los sistemas de trasplante manual (Lawyer, 1974).

Existen máquinas equipadas y automatizadas para realizar todas las tareas, hasta reducir al mínimo la participación del obrero, quien se limita a traer la plántula en charola y colocarla entre las pinzas sobre una corona circular que gira y que al llegar al suelo la libera automáticamente. Sin embargo, muchos programas de reforestación contemplan la participación del poseedor del bosque quien recibe remuneración monetaria. En estos casos, la tecnología también es importante, sobre todo para optimizar el tiempo y el esfuerzo necesarios para hacer las cepas (Keyes, 1996).

Por otra parte, el uso de tecnología no solamente puede mejorar el costo-beneficio de los proyectos sino interesar al usuario a cualquier nivel socio-económico. Para abrir el surco tras el paso de maquinaria agrícola, se logra minimizar la parte más laboriosa y desalentadora de la plantación. Hay otras tecnologías intermedias para apoyar las plantaciones forestales; otra variante del método manual de plantación con envase es la cava-hoyos que fue utilizada para fines comparativos. Ahora que los días de la economía cerrada en la mayor parte de los países de América Latina han terminado, hoy en día todavía es más necesario que se realicen inversiones a largo plazo para modernizar el equipamiento de los sectores productivos con el fin de hacer crecer sus respectivas capacidades y así operar a mayores escalas (Weiss, 1994).

\section{CONCLUSIONES}

En la hipótesis nula se esperaban mayores crecimientos de las especies en el sistema manual en donde hay mayores cuidados en cada hoyo excavado. Los métodos semi-mecanizados resultaron ser más baratos que los manuales y más eficientes en cuanto a tiempo en el presente ensayo. La superioridad del sistema mecanizado se demuestra tanto en los criterios económicos como en los aspectos de crecimiento de planta en La Chontalpa, Tabasco, México. Por consiguiente, al encontrar menos costos y mayores crecimientos en la plantación semimecanizada con el análisis estadístico, se tiene una pauta para rechazar esta hipótesis. La plantación semi-mecanizada o mecanizada en general puede resolver muchas dificultades cuando la escala de operaciones y el capital lo permita; sin embargo al parecer, plantaciones mecanizadas y semi-mecanizadas no constituyen ninguna panacea. El uso eficiente de recursos tecnológicos y de material vegetal con recursos humanos entrenados, sí permitiría mayores logros en extensión, tan necesarios para enfrentar el reto de la reforestación en el trópico. El uso de maquinaria disponible con pocas modificaciones de acuerdo con las características del sitio, podría mejorar la eficiencia tanto en tiempo como en mano de obra y también en costos.

\section{REFERENCIAS}

Cannon, P. 1982. Ensayo de preparación de sitio en suelos volcánicos de Pinus y Eucalyptus: resultados después de tres años. Informe de Investigación No. 75. Smurfit Cartón de Colombia, S.A. 8p.

Cannon, P., J. Idarraga y L. León. 1984. Uso de herbicidas para controlar la competencia de pastos en plantaciones 
forestales. Informe de Investigación. No. 90. Smurfit Cartón de Colombia. 14p.

Chapman, G. y T. Allen. 1978. Establishment techniques for forestry plantations. FAO Forestry Paper No. 18 FAO, Roma.

Del Castillo C., P. 1996. Evaluación financiera sobre plantaciones comerciales. Madera y Bosques 2(1): 3-8.

Daniel, J., J. Helms y F. Baker. 1982. Principios de Silvicultura. McGraw-Hill, México, D.F.

Endean, F. y D. Hocking. 1973. Performance after planting of four types of container-grown Lodgepole pine seedlings. Canadian Journal of Forest Research 3(2): 185-195.

Endo, M. 1992. Resultados del primer año del proyecto de la Costa Norte en Monterrubión y San Sebastián. Informe de Investigación No. 143. Smurfit Cartón de Colombia. 21p.

Endo, M. y J. Wright. 1992. Crecimiento de una plantación de Eucalyptus grandis bajo diferentes niveles de control de malezas. Informe de Investigación No. 148. Cartón de Colombia. 12p.

Evans, J. 1982. Plantation forestry in the tropics. Claredon Press. Oxford, England.

Keyes, M. 1996. Tecnología para la reforestación en América Latina. Madera y Bosques 2(1): 63-76.

Lamberth, C. 1986. El control de pastos con el herbicida Roundup aumenta los rendimientos de Eucalyptus globulus en la finca Salinas. Informe de
Investigación No. 108. Smurfit Cartón de Colombia. $4 p$.

Ladrach, W. 1982. Preparación mecánica y química del sitio para la reforestación de pendientes en grama; resultados después de un año. Informe de Investigación No. 78. Smurfit Cartón de Colombia, S.A. 4p.

Lawyer, J. 1974. Review of the literature on mechanized planting systems. pp. 29-38 In: Lawyer, J., Ed. Chapter 4. Analysis of mechanized systems for planting trees for reforestation. Dept. of Agricultural Engineering, University of California, Davis, California.

McKenzie, D., D. Hotfield, y K. Kyman. 1984. Tree-planting machines- can you afford one? Tree Planters' Notes 2: 8-11.

McNabb, D., K. Baker-Katz y S. Tesch. 1993. Machine site preparation improves seedling performance on a high-elevation site in southwest Oregon. Southern Journal of Applied Forestry. 8(3): 95-98.

Osorio, L. 1988. Preparación físico y químico de una área en potreros para la reforestación con Eucalyptus grandis, Cupressus lusitanica, Pinus oocarpa resultados a los cinco años. Informe de Investigación No. 118. Smurfit Cartón de Colombia. 10p.

Powers, R., W. Webster y P. Cochran. 1988. Estimating the response of Ponderosa pine forests to fertilization. pp. 219-226 In: Proc. Future forests of the mountain West: A stand culture symposium. USDA For. Serv. Gen. Tech. Rep. INT. 243. 
Retrepo, G. 1990. Preparación de terreno para plantación de Tabebuia rosea y Eucalyptus tereticornis $\mathrm{p} 99 . \mathrm{In}$ : Proceedings of the World Bank Annual Conference on Development Economics. 1990. The environment and emerging development issues. World Bank. Washington D.C.

Richardson, K. 1989. Forester's farm trees to meet growing timber needs on flat sites. Shell Agriculture. No. 25-26.

Rojas, G. 1995. Experiencias de plantación comercial de Cedrela odorata en sistemas agroforestales en la región de Los Tuxtlas, Veracruz. Tesis de Ingeniero Agrónomo Especialista en Bosques. Universidad Autónoma Chapingo. 114p.

Sánchez, S., M. Keyes, A. Trinidad H. y V. Cetina. 1988. Comparación de tres técnicas de producción en vivero de 15 especies de pinos. Agrociencias 72:9-26.

Trujillo, A. y D. Palma. 1988. Programa del uso del suelo. Estudio ecológico detallado del CEICADES. Colegio de Posgraduados. Cárdenas, Tabasco. Inédito.

USFS. 1967. List of Commercial Tree Planters. Tree Planters' Notes 1:18-21.

Weiss, C. 1994. Technological modernization in Latin America. Interciencia 19: 229-238.

Zanartu, E. 1968. Mecanización de plantaciones forestales en zonas de arenales. Actas Cuartas Jornadas Forestales. Asociación Chilena de Ingenieros Forestales. Valparaíso, Chile. $53 p$. 